\title{
The Dilemma of Coexisting Nevoid Hyperkeratosis of the Nipple and Areola in Mycosis Fungoides: A Report of Three Cases
}

\author{
Algun Polat Ekincia ${ }^{a}$ Sule Ozturk Sari ${ }^{b} \quad$ Nesimi Buyukbabani ${ }^{b}$ \\ Can Baykal ${ }^{a}$ \\ Departments of a Dermatology and Venereology and ${ }^{b}$ Pathology, Istanbul Medical Faculty, \\ Istanbul University, Istanbul, Turkey
}

Key Words

Nevoid hyperkeratosis of the nipple and the areola $\cdot$ Mycosis fungoides

\section{Abstract}

Nevoid hyperkeratosis of the nipple and areola (NHNA) is a rare clinicopathological entity showing persistent and strictly localized hyperkeratotic lesions of the nipple, areola or both with unknown etiopathogenesis. A similar clinical appearance may also be seen in different diseases with specific histopathological features. There are a few anecdotal reports on the association of NHNA with mycosis fungoides (MF), but they do not describe a uniform condition. In this report, we present 3 patients with hyperkeratotic lesions of the nipple and areola associated with MF but showing different histopathological features. We also review similar cases in the literature and discuss possibilities concerning this association. Two of our cases represent the association between MF and NHNA without histopathological features of MF on the nipple-areola complex. The other case represents hyperkeratosis of the nipple and areola with specific histological and immunohistochemical features of MF. Hence, we would like to hypothesize that MF may involve the nipple and areola and have an appearance similar to NHNA. Intriguingly, however, NHNA may occasionally also be seen in association with MF. However, this peculiar association requires further explanation. 
Polat Ekinci et al.: The Dilemma of Coexisting Nevoid Hyperkeratosis of the Nipple and Areola in Mycosis Fungoides: A Report of Three Cases

\section{Introduction}

Nevoid hyperkeratosis of the nipple and areola (NHNA) is a rare clinicopathological entity including persistent and strictly localized hyperkeratotic lesions of the nipple, areola or both $[1,2]$. The etiopathogenesis of this benign condition is unknown [2]. In 1938, Levy-Franckel [3] classified hyperkeratosis of the nipple and areola into three categories: (1) as an isolated or nevoid form, (2) as an extension of the epidermal nevus or (3) in association with ichthyosis or other skin conditions. However, these latter diseases, characterized by secondary involvement of the nipple-areola complex, typically exhibit specific histopathological features and are infrequently restricted to this area. Hence, the practical significance of this classification is limited. In 1990, Pérez-Izquierdo et al. [4] classified NHNA from an etiological viewpoint into nevoid (idiopathic) and secondary variants. Though acanthosis nigricans, verrucous nevus, seborrheic keratosis, dermatosis, ichthyosis, lymphoma, Darier's disease, eczema and drug reactions were cited as secondary causes of NHNA in this classification, a pathogenetic mechanism for these associations was not proposed. There have been anecdotal reports of an association between NHNA and mycosis fungoides (MF) [5-8], and of cases mimicking MF and exhibiting histopathological changes such as intraepidermal collections of lymphocytes $[8,9]$.

We evaluated the data on 565 registered patients in our outpatient clinic for MF patients. Hyperkeratotic lesions of the nipple and areola were observed in 3 patients with MF elsewhere. Herein, we present these 3 MF cases displaying an association with hyperkeratotic lesions of the nipple and areola but showing different histopathological features. We also review similar cases in the literature and discuss possibilities regarding this relationship.

\section{Case Reports}

Case 1

A 31-year-old male presented with erythematous patches and plaques on the trunk, as well as asymptomatic brownish thickening of the skin of the right nipple and areola, which he had had for several years (fig. 1a). Histological examination of a plaque on the trunk confirmed a diagnosis of MF; examination of a nipple lesion revealed hyperkeratosis, keratotic plugs, acanthosis and papillomatosis, without associated epidermal or dermal inflammatory or neoplastic infiltration (fig. 1b), clearly suggesting NHNA. The patient was treated with PUVA for MF, with topical retinoic acid for NHNA. The MF lesions improved completely after 1 year of therapy, but a prominent response was not observed for the nipple lesion.

\section{Case 2}

A 62-year-old male presented with MF relapse characterized by erythematous plaques on the trunk and arms and hyperkeratotic lesions of the nipple-areola complex. New skin lesions on the trunk had appeared during the previous 2 years, with bilateral areolar lesions having shown during the previous 3 months. Hyperkeratosis was prominent on the areola, but the nipple was spared (fig. 2a). Areola biopsy revealed only epidermal changes such as profound hyperkeratosis, mild acanthosis and papillomatosis, confirming the NHNA diagnosis (fig. 2c, d). The patient was treated with PUVA for MF and had no specific treatment for the nipple lesions. The MF plaques healed completely following 12 months of therapy, and the areolar hyperkeratosis decreased markedly (fig. 2b).

Case 3

An 80-year-old male presented with disseminated erythematous patches, plaques and erosions (fig. 3a) on the entire body. Furthermore, there was infiltration covered with hyper- 
Polat Ekinci et al.: The Dilemma of Coexisting Nevoid Hyperkeratosis of the Nipple and Areola in Mycosis Fungoides: A Report of Three Cases
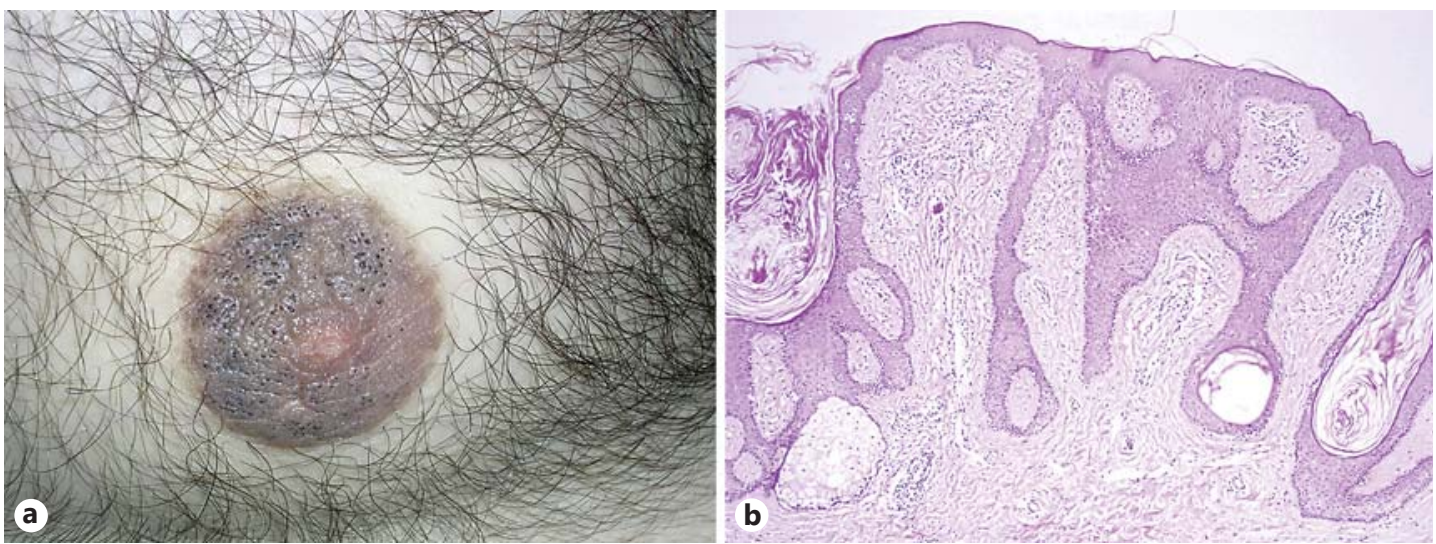

Fig. 1. a Diffuse hyperkeratosis of the nipple and areola. Keratin pearls are also noticeable on the surface. b Slight hyperkeratosis, follicular plugging, acanthosis and prominent papillomatosis. There is no neoplastic infiltration in the papillary dermis. HE. $\times 100$.
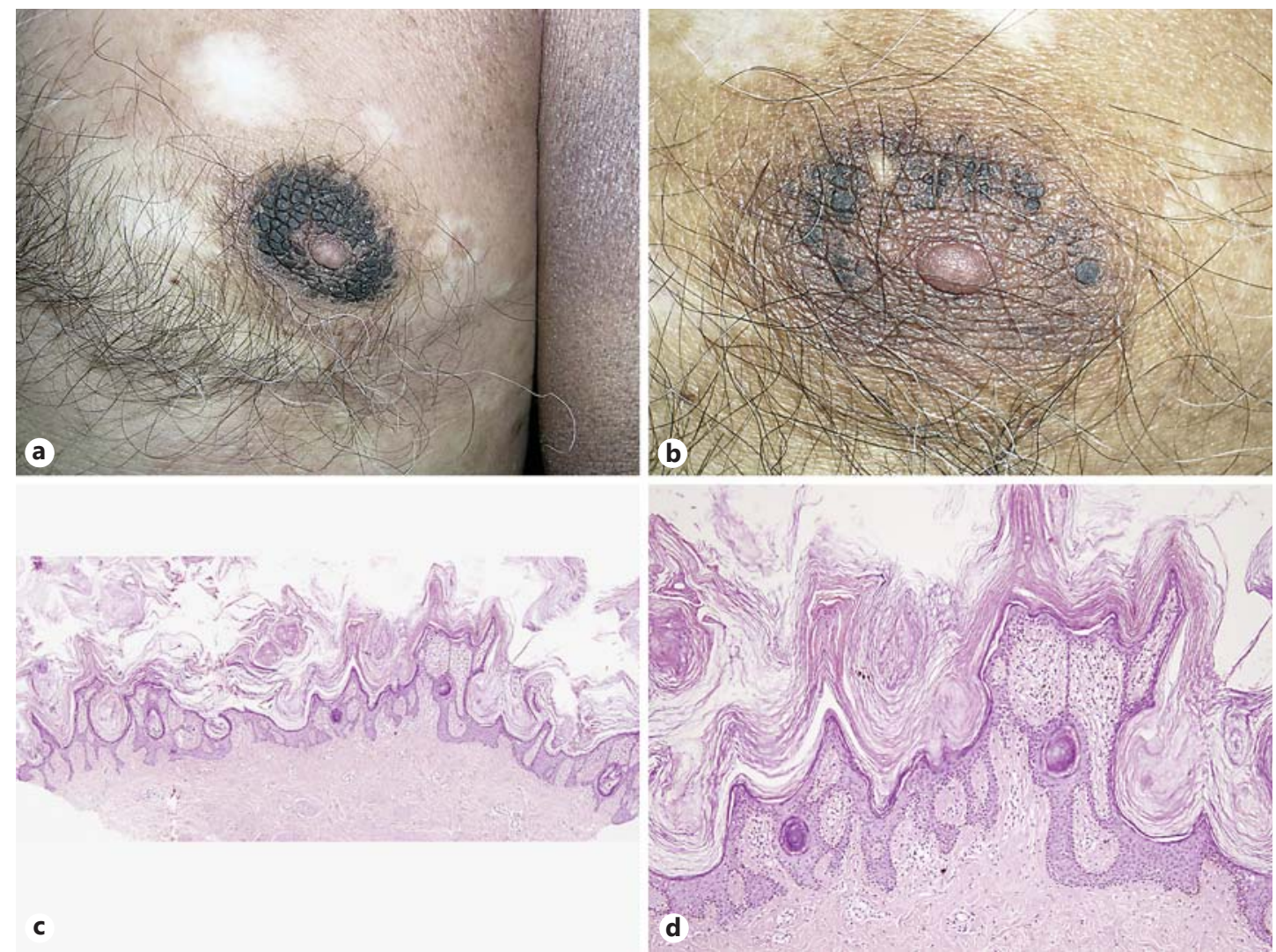

Fig. 2. a Dark brown verrucous thickening of the areola associated with plaques of MF on the upper arm. b Substantial improvement of the hyperkeratosis of the areola after PUVA therapy for MF. c, d Histopathology shows very prominent hyperkeratosis, verruciform acanthosis and papillomatosis. There is no infiltration in the papillary dermis. HE. $\mathbf{c} \times 40 . \mathbf{d} \times 100$. 

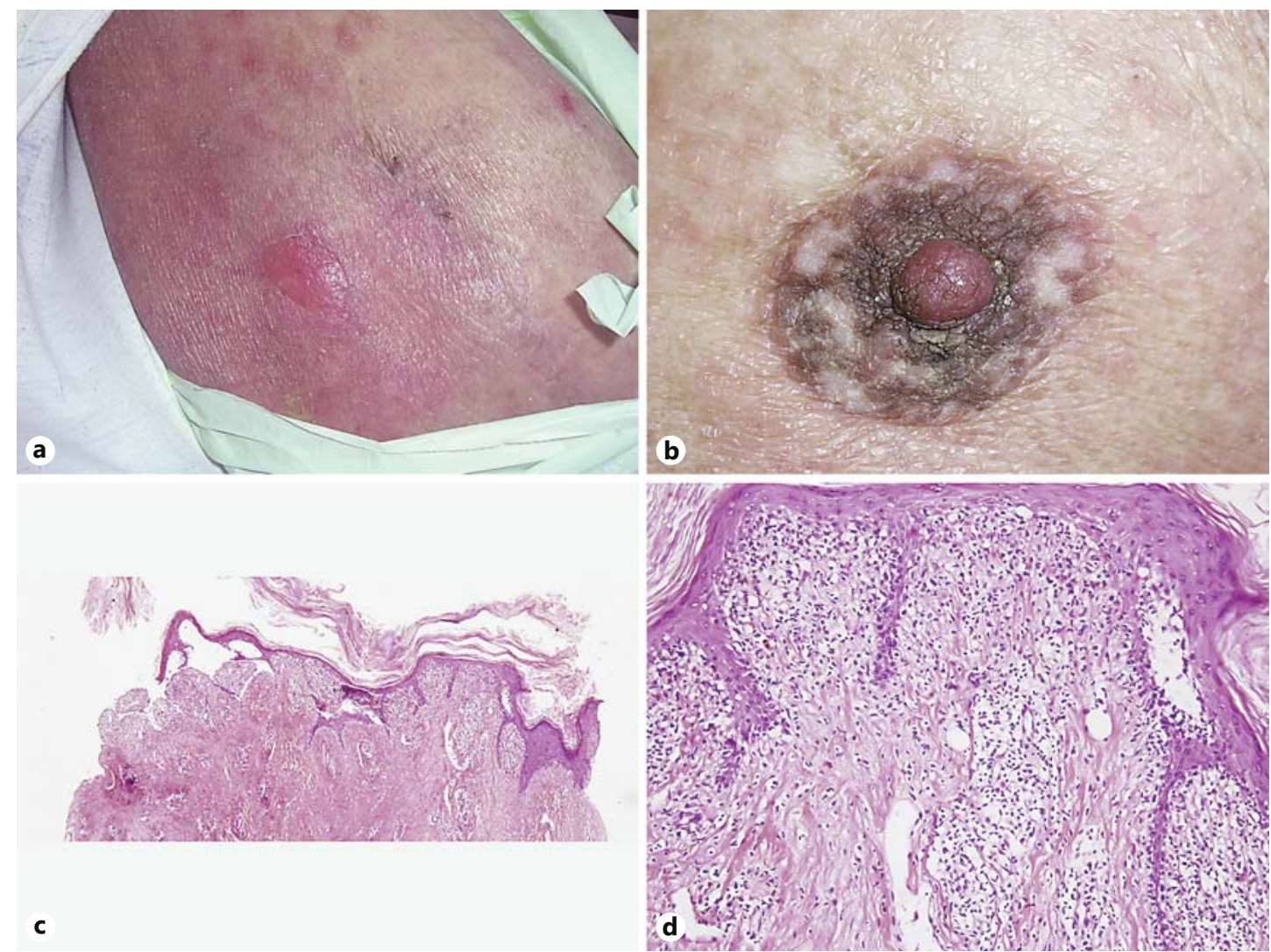

Fig. 3. a Erythematous plaques and an erosion on the thigh representing bullous MF. b Infiltrated plaque covered with hyperkeratosis located on the nipple and areola. c, $\mathbf{d}$ Hyperkeratosis is prominent. The epidermis is thinned and separated from the dermis due to biopsy trauma. There are numerous lymphocytes in the junctional area, which were interpreted as MF involvement of the areola. HE. $\mathbf{c} \times 40 . \mathbf{d} \times 200$.

keratosis on the areola, bilaterally (fig. 3b). Biopsy of an eroded area on the trunk revealed bullous MF. A direct immunofluorescence examination was negative. Histopathological examination of the right areola revealed typical MF infiltration characterized by the presence of CD4-positive cells, a remarkably elevated ratio of CD4/CD8 and complete loss of CD7 within the papillary dermis, as well as marked epidermotropism associated with other epidermal changes such as orthokeratotic hyperkeratosis (fig. 3c, d). The patient, who had multiple comorbidities, was treated with methotrexate for MF but could not tolerate the therapy.

\section{Discussion}

Hyperkeratotic lesions of the nipple and areola were observed in 3 of 565 patients with MF. Typically, NHNA lesions are characterized by persistent verrucous thickening and dark brown pigmentation of the nipple and areola [1], with no induration or discharge [2]. These clinical features were observed in all 3 cases, but case 3 was further characterized by infiltration on palpation. Clinicopathological correlation is important for the diagnosis of NHNA. Epidermal changes such as orthokeratotic hyperkeratosis, acanthosis, keratin plugging, anastomosing rete ridges and the striking filiform nature of papillomatosis represent the main histopathological features of NHNA [2]. Although these histopathological features may mimic those of epidermal 
nevus or acanthosis nigricans, lesions of NHNA are only regional and there is no association with any systemic disease. All of our 3 cases have widely distributed patches or plaques of MF, which was confirmed by biopsy, and also hyperkeratosis on the nipple and areola. Histopathological examination of the nipple or areola specimens revealed typical MF features in addition to hyperkeratosis and acanthosis in only 1 case, and features of NHNA alone in the remaining 2 cases.

In the classification system of NHNA proposed by Pérez-Izquierdo [4], among other dermatologic diseases lymphoma was also considered as causing secondary involvement of the nipple-areola complex. On the other hand, only a few well-documented cases have been reported pertaining to this relationship, which do not appear to describe a uniform condition [5-9]. According to a review addressing the clinicopathological spectrum of MF, lesions of vegetating/papillomatous MF may resemble acanthosis nigricans or seborrheic keratosis which can be seen on the nipple and areola [10]. Very prominent papillomatous and verrucous lesions in an MF patient were first reported by Kanitakis and Tsoitis [5] in 1977. Allegue et al. [6] described hyperkeratosis of the nipple and areola in a patient with cutaneous T-cell lymphoma. Furthermore, Ahn et al. [7] described another case characterized by simultaneous, generalized skin eruption and hyperkeratotic verrucous nipple lesions exhibiting additional histopathological features, including atypical lymphoid infiltration in the dermis.

An atypical case of bilateral NHNA, characterized by histopathological features resembling MF but without any other MF lesions on the body, was described by Roustan et al. [8]. An exceptional case of unilateral NHNA, exhibiting intraepidermal lymphocytes resembling Pautrier's microabscesses, has also been reported [9]. In the latter case, the intraepidermal lymphocytes demonstrated CD3 positivity, CD4 predominance and CD7 loss but no clonal $\mathrm{T}$-cell gene rearrangement during molecular analysis. The lesions remained stable over several years without development of cutaneous or systemic manifestations of lymphoma. Unilateral hyperkeratosis of the nipple and areola was reported as the initial lesion of MF in a child [11]. In this case, the immunohistochemical and T-cell gene rearrangement studies of the intraepidermal lymphocytes were consistent with MF.

In accordance with previous reports in the literature, and the 3 novel cases, we would like to hypothesize the following. (1) MF patients diagnosed histologically with lesions of other parts of the body may be said to display an association with hyperkeratosis of the nipple and areola with specific histological infiltration of MF (such as in case 3). (2) MF patients diagnosed histologically with lesions of other parts of the body may indicate NHNA without histological features of MF (such as in cases 1 and 2). Because NHNA is known as a very rare disease, this peculiar association requires further explanation. Another possibility is that NHNA is an underestimated condition and more easily diagnosed in patients with MF, who are frequently exposed to total body examination. (3) Several previously reported cases indicated that isolated hyperkeratotic lesions of the nipple and areola exhibited histological features of MF in addition to the classic epidermal features of NHNA [8, 9], possibly indicating 'unilesional MF or early lesion of MF' in unusual locations [11]. As all of the cases had histologically confirmed MF lesions in other areas, none of them could be included in this group.

\section{Statement of Ethics}

Legally effective written informed consent has been obtained from each patient.

\section{Disclosure Statement}

The authors declare no conflict of interest. No funding has been obtained for this work. 
Polat Ekinci et al.: The Dilemma of Coexisting Nevoid Hyperkeratosis of the Nipple and Areola in Mycosis Fungoides: A Report of Three Cases

\section{References}

1 Baykal C, Buyukbabani N, Kavak A, Alper M: Nevoid hyperkeratosis of the nipple and areola: a distinct entity. J Am Acad Dermatol 2002;46:414-418.

2 Krishnan RS, Angel TA, Roak TR, Hsu S: Nevoid hyperkeratosis of the nipple and areola: a report of two cases and a review of the literature. Int J Dermatol 2002;41:775-777.

3 Levy-Franckel A: Les hyperkératoses de l'aréole et du mamelon. Paris Med 1938;28:63-66.

4 Pérez-Izquierdo JM, Vilata JJ, Sánchez JL, Gargallo E, Millan F, Aliaga A: Retinoic acid treatment of nipple hyperkeratosis. Arch Dermatol 1990;126:687-688.

5 Kanitakis C, Tsoitis G: Mycosis fungoides and follicular mucinosis with very prominent papillomatous and verrucous lesions (in French). Dermatologica 1977;155:268-274.

6 Allegue F, Soria C, Rocamora A, Fraile G, Ledo A: Hyperkeratosis of the nipple and areola in a patient with cutaneous T-cell lymphoma. Int J Dermatol 1990;29:519-520.

7 Ahn SK, Chung J, Soo Lee W, Kim SC, Lee SH: Hyperkeratosis of the nipple and areola simultaneously developing with cutaneous T-cell lymphoma. J Am Acad Dermatol 1995;32:124-125.

8 Roustan G, Yus ES, Simón A: Nevoid hyperkeratosis of the areola with histopathological features mimicking mycosis fungoides. Eur J Dermatol 2002;12:79-81.

9 Rosman IS, Hepper DM, Lind AC, Anadkat MJ: Nevoid hyperkeratosis of the areola misinterpreted as mycosis fungoides. J Cutan Pathol 2012;39:545-548.

10 Kazakov DV, Burg G, Kempf W: Clinicopathological spectrum of mycosis fungoides. J Eur Acad Dermatol Venereol 2004;18:397-415.

11 Yalçın B, Gür G, Tabanlığlu-Onan D, Ekici Ö: Mycosis fungoides mimicking nevoid hyperkeratosis of the nipple and areola in an adolescent. Turk J Pediatr 2014;56:565-567. 